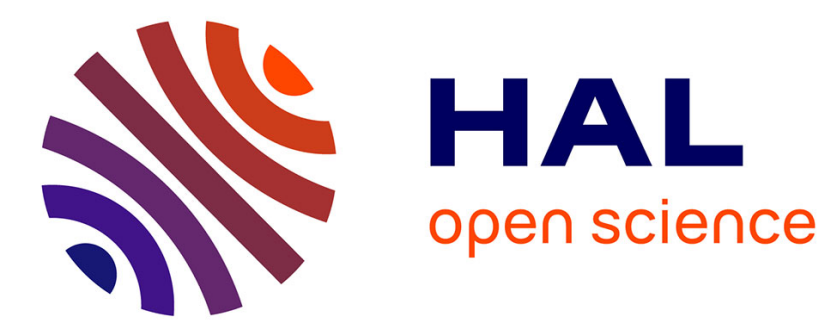

\title{
Parameter estimation for the characterization of thermoacoustic stacks and regenerators
}

Matthieu Guédra, Flavio Bannwart, Guillaume Penelet, Pierrick Lotton

\section{To cite this version:}

Matthieu Guédra, Flavio Bannwart, Guillaume Penelet, Pierrick Lotton. Parameter estimation for the characterization of thermoacoustic stacks and regenerators. Applied Thermal Engineering, 2015, 80, pp.229-237. 10.1016/j.applthermaleng.2015.01.058 . hal-02057305

\section{HAL Id: hal-02057305 \\ https://hal-univ-lemans.archives-ouvertes.fr/hal-02057305}

Submitted on 5 Mar 2019

HAL is a multi-disciplinary open access archive for the deposit and dissemination of scientific research documents, whether they are published or not. The documents may come from teaching and research institutions in France or abroad, or from public or private research centers.
L'archive ouverte pluridisciplinaire HAL, est destinée au dépôt et à la diffusion de documents scientifiques de niveau recherche, publiés ou non, émanant des établissements d'enseignement et de recherche français ou étrangers, des laboratoires publics ou privés. 


\title{
Parameter estimation for the characterization of thermoacoustic stacks and regenerators
}

\author{
Matthieu Guédra ${ }^{\mathrm{a}, 1, *}$, Flavio C. Bannwart ${ }^{\mathrm{a}}$, Guillaume Penelet $^{\mathrm{a}}$, Pierrick Lotton ${ }^{\mathrm{a}}$ \\ ${ }^{a}$ LUNAM Université, Laboratoire d'Acoustique de l'Université du Maine - UMR CNRS 6613, Avenue \\ Olivier Messiaen, 72085 Le Mans Cedex 9, France
}

\begin{abstract}
This paper deals with the in-situ characterization of open-cell porous materials that might be used as a so-called stack (or regenerator) in a thermoacoustic engine. More precisely, the manuscript presents an inverse method aiming at estimating geometrical and thermal properties of various samples of porous media surrounded by heat exchangers and connected to a thermal buffer tube to form a ThermoAcoustic Core (TAC). This estimation is realized from acoustic measurements, and it is expressed as a minimization problem applied to the squared norm of the difference between experimental and theoretical transfer matrices of the TAC. Experimental data, obtained for different stacks (ceramic catalyst, pile of stainless steel wire meshes, carbon and metallic foams) under various heating conditions, are used in order to fit the theoretical forward model by adjusting geometrical properties of the sample and heat exchange coefficients. Common geometrical properties (porosity and average pore's radius) obtained with the present method are consistent with available data from manufacturers. Moreover, this method allows to estimate the tortuosity of the material which is not given by manufacturers. Estimation of heat coefficients (and their variations with heating) provides global information about anisotropic heat diffusion through the porous material employed as a thermoacoustic stack submitted to a temperature gradient. Among the four characterized samples, it appears that the carbon foam allows to get the highest temperature gradients
\end{abstract}

\footnotetext{
*Corresponding author : matthieu.guedra@dalembert.upmc.fr

${ }^{1}$ Current address: Université Pierre et Marie Curie, Institut Jean Le Rond d'Alembert - UMR CNRS 7190, 4 place Jussieu, 75252 Paris cedex 05, France 
and thus can be considered as the most efficient regenerator for energy conversion in a thermoacoustic prime mover.

Keywords: Thermoacoustics; Inverse Problem; Parameter estimation; Porous materials

\section{Introduction}

Thermoacoustic prime-movers are specific kinds of heat engines, which basically consist of a porous medium, referred to as a stack or a regenerator, inserted into an acoustic resonator. The operation of such a system consists in applying a steep temperature gradient along the stack, which leads to the onset of self-sustained acoustic oscillations at the frequency of the most unstable acoustic mode of the resonator. The resulting mechanical (acoustical) work can be used for the production of electricity[1] or for thermoacoustic refrigeration [2]. Thermoacoustic engines have fundamental advantages such as low maintenance costs, simplicity, and they are able to achieve good efficiency. Potential applications of thermoacoustic engines include waste heat recovery $[3,4,5]$ or solar powered thermodynamic engines $[6,7]$.

The operation of a thermoacoustic system - and especially the onset of self-sustained waves in thermoacoustic prime movers - depends mainly on the thermal exchanges involved in the stack and the heat exchangers. In the early versions of thermoacoustic engines built in the mid 90's, the thermal interaction between the fluid and the solid was realized by using porous materials of simple geometry such as stacks of parallel plates $[8,9]$ or honeycombed materials [10]. However, recent research works have shown that the increase of efficiency of thermoacoustic systems may involve using more "exotic" materials, such as pin-array stacks [11], Reticulated Vitreous Carbon (RVC) foams [12] or Stainless Steel wire meshes [13], which are clearly anisotropic materials.

The theoretical description of the porous elements in the active thermoacoustic cell is essential for the design of thermoacoustic systems. The standard periodic frames such as parallel plates or uniformly-shaped channels networks may be described with the capillary-tube-based thermoacoustic theory $[14,15,16,17]$. In this theory, the viscous and thermal functions $f_{\nu}$ and $f_{\kappa}$ - whose analytical expressions depend on the geometry of the channel - are introduced for taking into account the coupling between the oscillating fluid and the solid medium. In 1991, Roh et al. [18] notably validated with 
experiments Stinson's theoretical work for rectangular pores [17]. Numerous works have been made since then, in order to expand the theory to more complex porous frames. In a series of papers [19, 20,21], Wilen and Petculescu measured the thermoviscous functions of different materials (notably RVC foams and aluminum foams), with and without temperature gradient. In 2005, Muelheisen et al. [22] used a 4-microphones method in order to measure the characteristic impedance and the complex wave number of RVC foams, and compared their results with those obtained with semi-analytical $[23,24]$ and analytical [25] models of porous media. In recent works, Roh et al. developed a mathematical model for inhomogeneously heated porous materials [26], based on the capillary-based thermoacoustic theory, which showed good agreement with the experimental results obtained by Wilen and Petculescu [20,21], with an adjustement of shape factors $n_{\nu}$ and $n_{\kappa}$, together with the tortuosity $q$ of the material.

However, as do the geometrical properties of the stack/regenerator, the temperature distribution strongly affects the operation of a thermoacoustic system [27]. Therefore, an accurate description of the onset and amplification processes governing the thermoacoustic instability should involve considering an accurate modeling of heat transfer in each element constituting the ThermoAcoustic Core (TAC). This accurate description remains arduous as it implies that the thermophysical properties - e.g. the axial and transverse thermal conductivities - of the anisotropic stacks or regenerators are known. In this context, our objective in this paper is to use an inverse method allowing to fit a theoretical model describing acoustic propagation in the inhomogeneously heated TAC with experiments, and to estimate geometrical and thermal parameters of the porous material.

In previous studies $[28,29]$, we presented an experimental setup for the measurements of the acoustical transfer matrix of a TAC under various heating conditions, and we used the experimental data to predict the onset of self-sustained oscillations of any thermoacoustic device equipped with the TAC characterized beforehand. However, such a black box approach does not provide information about the inside of the thermoacoustic core, and therefore the main objective of this study is to evaluate an inverse method approach to get information about the acoustical and thermal properties of the stack/regenerator. More precisely, the purpose of the present paper is (1) to propose a theoretical description 

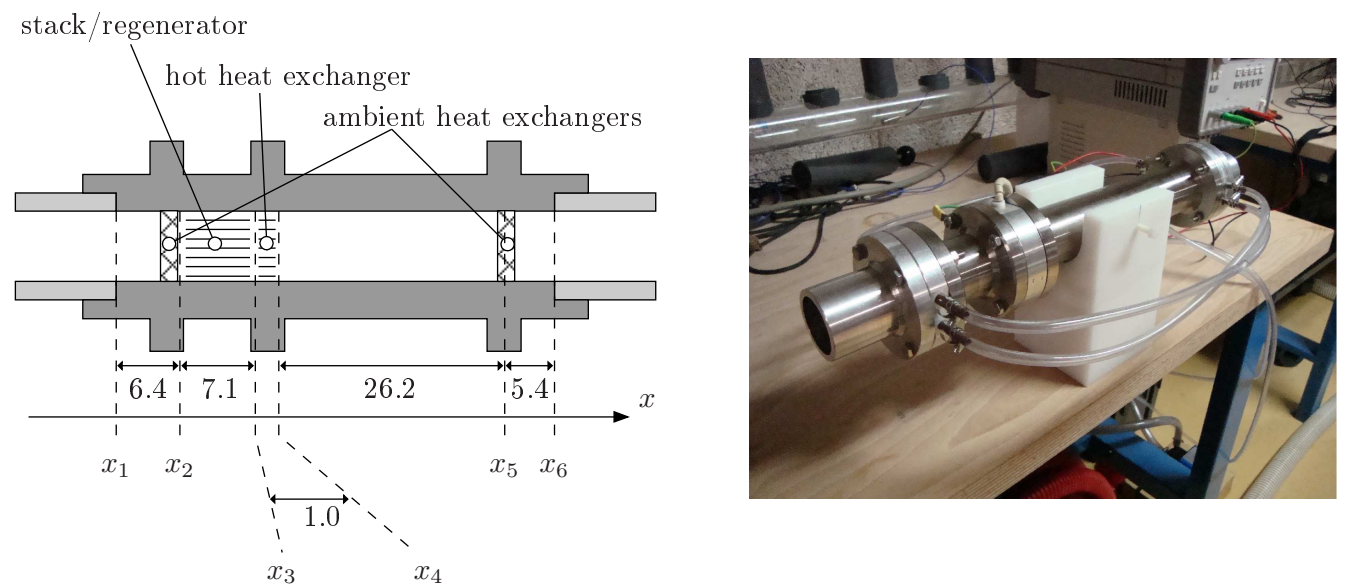

Figure 1: Schematic drawing and photograph of the TAC. Lengths are given in $\mathrm{cm}$.

of the TAC and (2) to make use of the experimental results from [28, 29] so as to adjust parameters which are generally hard to evaluate (tortuosity, heat exchange coefficient ... ). In section 2, a brief description of the TAC under study is presented. In section 3 , we describe the theoretical network modelling of the TAC with non-constant temperature gradients. The inverse method used to fit this model with experimental data obtained in the previous studies is presented in section 4 and the results of the parameter estimation are presented and discussed in section 5 : this method allows notably to estimate the porosity and the tortuosity of the porous sample, as well as the average inner radius of the pores and the heat exchange coefficients with the walls.

\section{Description of the ThermoAcoustic Core and T-matrix measurements}

The TAC under study is shown in Fig. 1. In the following, we give a brief description of this element and of the experimental procedure used for measuring its transfer matrix (for a complete description of the measurements methods, the reader can find more details in Refs. $[28,29]$ ). The TAC consists of a cylindrical waveguide (inner radius $R=$ $16.7 \mathrm{~mm}$ ) compounded of several aluminum and Stainless Steel pieces. Two honeycombed ambient exchangers are used to remove heat from the system. The hot exchanger is made of a piece of ceramic sample with squared pores of density 600 CPSI (Cells Per Square Inch). The heat power is supplied to the system by means of a Nickel-Chromium resistant wire which is regularly coiled across the whole cross section of the exchanger. 
The acoustic properties of the TAC can be characterized by means of the so-called transfer matrix [30] (T-matrix), which gives a relationship between the complex amplitudes of acoustic pressure and acoustic velocity at both sides of the TAC. More precisely, the T-matrix of the TAC is here defined as

$$
\left(\begin{array}{c}
\tilde{p}\left(x_{6}\right) \\
\tilde{u}\left(x_{6}\right)
\end{array}\right)=\left(\begin{array}{cc}
\mathcal{T}_{p p} & \mathcal{T}_{p u} \\
\mathcal{T}_{u p} & \mathcal{T}_{u u}
\end{array}\right) \times\left(\begin{array}{c}
\tilde{p}\left(x_{1}\right) \\
\tilde{u}\left(x_{1}\right)
\end{array}\right),
$$

where $\tilde{p}\left(x_{i}\right)$ and $\tilde{u}\left(x_{i}\right)$ denote the complex amplitudes of acoustic pressure and acoustic volume velocity at position $x_{i}$ (with $x_{i}=x_{1,6}$, see Fig. 1). These complex amplitudes are defined from the relation $\xi(x, t)=\Re\left(\tilde{\xi}(x) e^{-i \omega t}\right)$, where $\xi(x, t)$ denotes the fluctuations of either acoustic pressure $(\xi \equiv p)$ or acoustic volume velocity $(\xi \equiv u)$, $\Re$ denotes the real part of a complex number, and where $\omega$ stands for the angular frequency of acoustic oscillations. Therefore, acoustic propagation through the TAC is only characterized by the four complex elements $\mathcal{T}_{i j}$ of its matrix, which depend on the frequency of acoustic oscillations, on the geometrical and thermophysical properties of the stack and heat exchangers, and on the assigned temperature distribution along the TAC. In previous works, we performed the measurements of the T-matrices of various TAC by means of two different methods, namely a classical two-load method [28] and another method based on the use of an acoustic impedance sensor [29]. As a result, experimental data for different kinds of TAC equipped with different stack materials (ceramic catalyst, stainless steel grids, RVC and NiCr foams) are now available, which are obtained for different values of heat power supply in the frequency range $30 \mathrm{~Hz}-500 \mathrm{~Hz}$. These data can thus be used to fit a model for the acoustic propagation through the TAC and to estimate some of its physical parameters. In the following, we describe the model and the inverse method used for the estimation.

\section{Theoretical description of the TAC - the forward problem}

\subsection{Acoustic propagation}

The propagation of harmonic plane waves in the TAC is derived from the transfer matrices formalism, for which each element (namely the heat exchangers, the stack and the thermal buffer tube, see Fig. 1) is considered as an acoustical two-port. As they are made of honeycombed materials with quite large open cells $(\sim R / 10)$ and high 
porosity, the ambient exchangers are neglected by considering that they do not perturb the propagation of the plane wave, so that they can be considered as being part of the large waveguide.

When a heat power $Q$ is supplied to the hot exchanger, a temperature distribution $T(x)$ is established along the TAC, and sound propagation of plane waves along $x$ is described as the combination of five two-port elements (see Fig. 1). As a result, the T-matrix of the TAC is defined as the following product:

$$
\left(\begin{array}{cc}
\mathcal{T}_{p p} & \mathcal{T}_{p u} \\
\mathcal{T}_{u p} & \mathcal{T}_{u u}
\end{array}\right)=\mathbf{T}_{\mathbf{g}, \mathbf{r}} \times \mathbf{T}_{\text {tbt }}(T(x)) \times \mathbf{T}_{\mathbf{h}}(T(x)) \times \mathbf{T}_{\mathbf{s}}(T(x)) \times \mathbf{T}_{\mathbf{g}, \mathbf{l}},
$$

where the T-matrices $\mathbf{T}_{\mathbf{g}, 1}$ and $\mathbf{T}_{\mathbf{g}, \mathbf{r}}$ describe sound propagation at room temperature $\left(T(x)=T_{c}\right)$ through the two ducts of radius $R$ at both sides, namely from $x=x_{1}$ to $x_{2}$ and from $x=x_{5}$ to $x_{6}$, respectively. The T-matrices $\mathbf{T}_{\mathbf{s}}, \mathbf{T}_{\mathbf{h}}$ and $\mathbf{T}_{\mathbf{t b t}}$ are those of the stack, the hot exchanger and the thermal buffer tube, respectively, which depend on the inhomogeneous temperature distribution $T(x)$ along the TAC due to the heat power $Q$ supplied to the hot heat exchanger. If this temperature distribution is known, then it is possible to calculate the above mentioned T-matrices. In the following, we derive the governing equations from which these T-matrices can be determined. Considering a piece of stack along which the temperature distribution $T(x)$ is assigned, the extension of Rott's theory [14] to porous media proposed by Roh et al. [26] can be used to derive the relationship between the complex amplitudes of acoustic pressure and volume velocity. However, here, we use a simplified version of this theory which does not account for the so-called viscous and thermal dynamic shape factors, but which accounts for the tortuosity $q$ of the material. With this assumption, the thermoacoustic wave equation can be written as [see Eq. (52) in ref.[26]]:

$$
\rho \frac{d}{d x}\left[\frac{1-f_{\nu}}{\rho} \frac{d \tilde{p}}{d x}\right]+\frac{1}{T(x)} \frac{d T}{d x} \frac{f_{\kappa}-f_{\nu}}{1-\operatorname{Pr}} \frac{d \tilde{p}}{d x}+q^{2}\left(\frac{\omega}{c_{0}}\right)^{2}\left[1+(\gamma-1) f_{\kappa}\right] \tilde{p}=0,
$$

where $c_{0}$ is the adiabatic sound speed, $\rho$ is the mean density of the fluid, Pr is the Prandtl number, and $\gamma$ is the ratio of specific heats. The functions $f_{\nu}$ and $f_{\kappa}$ defined as

$$
f_{\nu, \kappa}=\frac{2}{(1-i) \frac{r}{\delta_{\nu, \kappa}}} \frac{J_{1}\left((1-i) \frac{r}{\delta_{\nu, \kappa}}\right)}{J_{0}\left((1-i) \frac{r}{\delta_{\nu, \kappa}}\right)}
$$


are the well-known functions [14] which characterize the viscous and thermal coupling between the oscillating fluid and the waveguide walls $\left(J_{n}\right.$ is the $n^{\text {th }}$ order Bessel function of the first kind), where $\delta_{\kappa}=\sqrt{\frac{2 \kappa}{\omega}}$ and $\delta_{\nu}=\sqrt{\operatorname{Pr}} \delta_{\kappa}$ stand for the frequency-dependent viscous and thermal acoustic boundary layer thicknesses ( $\kappa$ denotes the thermal diffusivity of the fluid), and where $r$ denotes the radius of the channel (or of the capillary tubes) which is (are) considered. Therefore it is considered here that any element of the TAC is either described as a piece of a cylindrical duct (ambiant heat exchangers and thermal buffer tube) or as a porous material made with many cylindrical channels (stack and hot heat exchanger). In this latter case, the complicated structure of the material (e.g. for a metallic foam) can be taken into account with its tortuosity, $q$. Moreover, it is also possible to express the acoustic volume velocity as a function of the axial gradient of acoustic pressure [see. Eq. (32) in ref [26]] as follows:

$$
\tilde{u}=-i \frac{\phi S}{q^{2}} \frac{1-f_{\nu}}{\rho \omega} \frac{d \tilde{p}}{d x}
$$

where $S=\pi R^{2}$ denotes the cross-sectional area of the duct and where $\phi$ stands for the porosity of the material ( $\phi<1$ for the stack and the heat exchangers, and $\phi=1$ elsewhere). Introducing equation (5) into equation (3) leads after some algebra to a second relation between $\tilde{p}$ and $\tilde{u}$ as follows:

$$
\frac{d \tilde{u}}{d x}=i \frac{\phi S \omega}{\rho c_{0}^{2}}\left[1+(\gamma-1) f_{\kappa}\right] \tilde{p}-\frac{1}{T(x)} \frac{d T}{d x} \frac{f_{\kappa}-f_{\nu}}{(1-\operatorname{Pr})\left(1-f_{\nu}\right)} \tilde{u} .
$$

Finally, from equations (5) and (6), it is straightforward to express the T-matrix of a very short piece of stack - with length $d x$ much lower than the acoustic wavelength $2 \pi c_{0} / \omega$ - which relates the pressure and volume velocity at position $(x+d x)$ to the same quantities at position $x$ :

$$
\begin{aligned}
& \tilde{p}(x+d x)=\tilde{p}(x)+i \frac{q^{2} \rho \omega d x}{\phi S} \tilde{u}(x) \\
& \tilde{u}(x+d x)=i \frac{\phi S \omega d x}{\rho c_{0}^{2}}\left[1+(\gamma-1) f_{\kappa}\right] \tilde{p}(x)+\left(1-\frac{T(x+d x)}{T(x)} \frac{f_{\kappa}-f_{\nu}}{(1-\operatorname{Pr})\left(1-f_{\nu}\right)}\right) \tilde{u}
\end{aligned}
$$

From these relations, it is therefore possible to calculate the five T-matrices defined in Eq. (2), just by connecting the appropriate number of elementary T-matrices characterizing propagation of acoustic waves through a small length $d x$ of each element (namely, the stack, the hot heat exchanger, the thermal buffer tube and the two large ducts at 
temperature $T_{c}$ ). It is worth mentioning that the impact of the temperature distribution appears explicitely in the above mentioned equations, but also implicitely via the dependance with temperature of the static density $\rho$, the sound speed $c_{0}$ and the thermoviscous functions $f_{\nu, \kappa}$. We should also note that some parameters of equations (7-8), as well as those in the definition of $f_{\nu, \kappa}$ in equation (4), are different depending on whether acoustic propagation through the stack, the hot heat exchanger or the Thermal Buffer Tube are considered. More precisely, we define these parameters as follows:

$$
\begin{array}{llll}
r=R, & \phi=1, & q=1, & \forall x \in\left[x_{1}, x_{2}\right], \\
r=r_{s}, & \phi=\phi_{s}, & q=q_{s}, & \forall x \in\left[x_{2}, x_{3}\right], \\
r=r_{h}, & \phi=\phi_{h}, & q=q_{h}, & \forall x \in\left[x_{3}, x_{4}\right] . \\
r=R, & \phi=1, & q=1, & \forall x \in\left[x_{4}, x_{5}\right], \\
r=R, & \phi=1, & q=1, & \forall x \in\left[x_{5}, x_{6}\right] .
\end{array}
$$

Finally, using such a finite difference description of acoustic propagation through each element, it is possible to calculate the T-matrix of the complete TAC under an assigned temperature distribution $T(x)$.

\subsection{Heat diffusion}

Computing the T-matrix of the TAC defined in equation (1) from equations (7-8) implies to calculate the temperature distribution $T(x)$ imposed by the heat source $Q$. To that purpose, a simplified one-dimensional model is considered for the description of steady-state heat transfer. Note that although it is clear that there may exist significant temperature gradients along the transverse direction, the assumption of a onedimensional temperature field is well justified since the description of acoustic propagation through the TAC is based on the assumption of plane pressure waves. Therefore, a more realistic description of the temperature field would also require, in the context of this study, to reconsider the description of acoustic propagation within the TAC.

A schematic drawing of heat transfer through the TAC is given in Fig. 2. The simplified description of heat transfer in such a system mainly lies on four assumptions. First, the heat conduction in the walls is discarded, assuming that they are equivalent to perfect thermal sinks. Second, heat is supposed to be supplied to the whole system through 


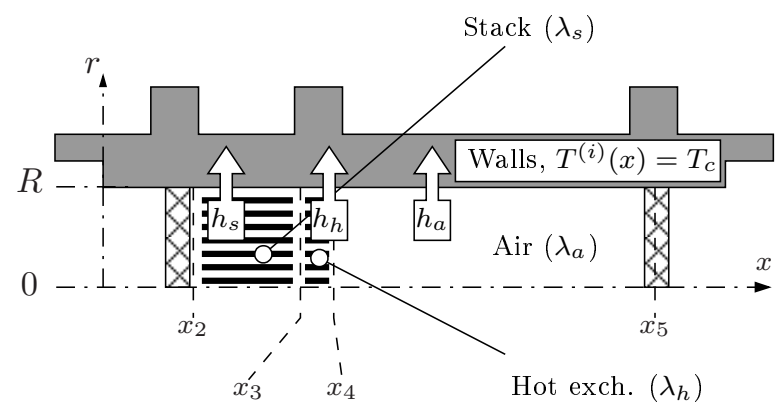

Figure 2: Schematic drawing of the TAC made of four separate media: the air in the thermal buffer tube, the stack/regenerator (considered as an equivalent homogeneous fluid medium), the hot exchanger (considered as an equivalent homogeneous fluid medium) and the stainless steel / aluminium walls.

a volumic heat source $Q$ dissipated in the total volume of the hot exchanger. Third, the ambient heat exchangers are discarded and we simplify the problem by assuming a zero heat flux at both sides. Finally, the thermal exchanges with the walls (radial heat leakages) are introduced by means of the equivalent thermal coefficients $h_{s}, h_{h}$ and $h_{a}$ (Newton's law for heat transfer), where the subscripts $s, h$ and $a$ are for the stack, the hot exchanger (both of them are considered as equivalent homogeneous fluid media) and the air in the thermal buffer tube, respectively.

Due to these assumptions, the steady-state temperature distribution can be described by three heat equations defined in the stack, in the hot heat exchanger and in the thermal buffer tube, respectively [31]:

$$
\begin{array}{ll}
\lambda_{s} \frac{d^{2} T}{d x^{2}}-\frac{2 h_{s}}{R}\left(T-T_{c}\right)=0, & \forall x \in\left[x_{2}, x_{3}\right], \\
\lambda_{h} \frac{d^{2} T}{d x^{2}}-\frac{2 h_{h}}{R}\left(T-T_{c}\right)=-\frac{Q}{l_{h} \pi R^{2}}, & \forall x \in\left[x_{3}, x_{4}\right], \\
\lambda_{a} \frac{d^{2} T}{d x^{2}}-\frac{2 h_{a}}{R}\left(T-T_{c}\right)=0, & \forall x \in\left[x_{4}, x_{5}\right],
\end{array}
$$

where $\lambda_{s}, \lambda_{h}$ and $\lambda_{a}$ denote the longitudinal conductivities of the stack, of the hot exchanger and of the air in the thermal buffer tube, respectively. Combining the set of heat diffusion equations (10) with appropriate junction conditions, namely a zero heat flux at boundaries,

$$
\left.\frac{d T}{d x}\right|_{x_{2}}=\left.\frac{d T}{d x}\right|_{x_{5}}=0
$$


and the continuity of temperature and heat flux at the interfaces,

$$
\begin{aligned}
T\left(x_{3}^{-}\right) & =T\left(x_{3}^{+}\right), \\
\left.\lambda_{s} \frac{d T}{d x}\right|_{x_{3}^{-}} & =\left.\lambda_{h} \frac{d T}{d x}\right|_{x_{3}^{+}}, \\
T\left(x_{4}^{-}\right) & =T\left(x_{4}^{+}\right), \\
\left.\lambda_{h} \frac{d T}{d x}\right|_{x_{4}^{-}} & =\left.\lambda_{a} \frac{d T}{d x}\right|_{x_{4}^{+}},
\end{aligned}
$$

the complete heat transfer model defined by Eqs. (10) and Eqs. (11)-(12) is finally solved using a finite differences scheme [33] to obtain the temperature distribution $T(x)$ along the TAC. Replacing this solution in Eq. (2) allows then to calculate the transfer matrix of the TAC with an imposed temperature gradient.

\section{The inverse problem}

Once the theoretical description of the TAC is derived, an inverse method can be used to fit the model with experimental data. The parameter estimation is performed with two steps. First, the acoustical model defined by Eq. (2) with $T(x)=T_{c}$ is considered and the experiments performed without heating are used to estimate the porosity, the tortuosity and the pore's inner radius of the porous material. Second, the model defined by Eq. (2) together with the thermal model derived in section 3.2 are considered and the experiments are used to estimate the heat exchange coefficients $h_{s}, h_{h}$ and $h_{a}$ in the various parts of the TAC. Such a strategy is well justified because the geometrical parameters do not depend on the temperature so that they can be estimated at first, without heating. As the inverse problem is intrinsically the same for the two steps mentioned above, the method is derived generally in this section.

The inverse problem consists in finding the parameters vector $\boldsymbol{\Upsilon}$ with $p$ elements (where $p$ is the number of parameters to estimate) which minimizes the squared norm $S$ of the difference between the experimental data and the theoretical transfer coefficients given by Eq. (2):

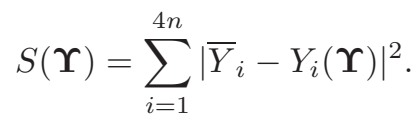

In Eq. (13), $n$ denotes the number of points on the frequency range, $\cdots$ denotes experimental data, and $\bar{Y}_{i}$ and $Y_{i}$ are the elements of the vectors obtained from a combination 
of the four T-matrix coefficients and defined as:

$$
\overline{\mathbf{Y}}=\left[\begin{array}{c}
\overline{\mathcal{T}}_{p p}\left(\omega_{1}\right) \\
\vdots \\
\overline{\mathcal{T}}_{p p}\left(\omega_{n}\right) \\
\overline{\mathcal{T}}_{p u}\left(\omega_{1}\right) \\
\vdots \\
\overline{\mathcal{T}}_{p u}\left(\omega_{n}\right) \\
\overline{\mathcal{T}}_{u p}\left(\omega_{1}\right) \\
\vdots \\
\overline{\mathcal{T}}_{u p}\left(\omega_{n}\right) \\
\overline{\mathcal{T}}_{u u}\left(\omega_{1}\right) \\
\vdots \\
\overline{\mathcal{T}}_{u u}\left(\omega_{n}\right)
\end{array}\right], \mathbf{Y}=\left[\begin{array}{c}
\mathcal{T}_{p p}\left(\omega_{1}\right) \\
\vdots \\
\mathcal{T}_{p p}\left(\omega_{n}\right) \\
\mathcal{T}_{p u}\left(\omega_{1}\right) \\
\vdots \\
\mathcal{T}_{p u}\left(\omega_{n}\right) \\
\\
\mathcal{T}_{u p}\left(\omega_{1}\right) \\
\vdots \\
\mathcal{T}_{u p}\left(\omega_{n}\right) \\
\\
\mathcal{T}_{u u}\left(\omega_{1}\right) \\
\vdots \\
\mathcal{T}_{u u}\left(\omega_{n}\right)
\end{array}\right]
$$

The minimization of the norm $S$ is a nonlinear parameter estimation problem [34] which involves using an iterative process to be solved. This is realized by calculating the parameters vector $\Upsilon$ at each step $(m+1)$ with the Levenberg-Marquardt's iterative relation [35]:

$$
\mathbf{\Upsilon}^{(m+1)}=\left[\mathbf{\Upsilon}+(\mathbf{H}+a \mathbf{I})^{-1} \Re\left(\mathbf{X}^{\dagger} \mathbf{e}_{\mathbf{Y}}\right)\right]^{(m)},
$$

where $\nmid$ denotes the adjoint operator, and where the vector $\mathbf{e}_{\mathbf{Y}}$ with $4 n$ elements corresponds to the prediction error, namely the difference between the measured and calculated values:

$$
e_{Y, i}=\bar{Y}_{i}-Y_{i}(\mathbf{\Upsilon})
$$

In Eq. (15), $\mathbf{X}$ is the sensitivity matrix of size $(4 n \times p)$ whose elements defined as:

$$
X_{i j}=\frac{\partial Y_{i}(\boldsymbol{\Upsilon})}{\partial \Upsilon_{j}} \quad \text { with } \quad i=1,2, \ldots 4 n \quad \text { and } \quad j=1,2, \ldots p,
$$

are calculated using a first-order finite-difference approximation. The pseudo-Hessian 
matrix $\mathbf{H}$ is then obtained from the sensitivity matrix $\mathbf{X}$ as follows:

$$
\mathbf{H}=\Re\left(\mathbf{X}^{\dagger} \mathbf{X}\right) .
$$

The steering factor $a$ in Eq. (15) allows to switch between the Gauss-Newton method $(a \rightarrow 0)$ and the Steepest Descent method $(a \rightarrow \infty)$. At the beginning of the iterations, $a$ is thus set to an arbitrary large value. At each iteration $m$, if $S^{(m)}<S^{(m-1)}$, the value of $a$ is divided by 2 in order to speed up the convergence with the GN method, otherwise it is multiply by 2 in order to take advantage of the robustness of the SD method and to enlarge the searching area.

Note that even though the minimization is realized on complex transfer coefficients, the matrices which are used in the iterative relation (15) are purely real and guarantee that the vector $\Upsilon$ is kept real at each step.

The iterative process is stopped when the relative variation of the parameters vector $\Upsilon$ is less than $0.1 \%$ from one step to the other. Moreover, as the convergence of the Levenberg-Marquardt method depends on the choice of the initial vector $\Upsilon^{(0)}$, a good $a$ priori knowledge of the actual values of the solution is required[36, 34]. To ensure the convergence, the inverse algorithm is also run a second time with a starting condition corresponding to a variation of $20 \%$ of the solution estimated at first time, in order to check that the same solution is reached.

The stability of the inverse method is controlled by the pseudo-Hessian matrix $\mathbf{H}$ which must be non-singular, i.e. the sensitivity coefficients should not be linearly dependent, and should be of the same order of magnitude [36, 34]. The stability of the algorithm has been checked by means of simulated experiments with artificial added noise and bias. For the acoustical problem (no heating), the estimation of the porosity, the tortuosity and the pore's inner radius of the stack/regenerator can be successfully achieved, but the estimation of a fourth parameter may make the algorithm unstable because an additional geometrical parameter would be correlated to the three above mentioned parameters (that is the reason why we did not account for the dynamic shape factors [26] in the description of acoustic propagation through the stack). 


\section{Results for parameter estimation}

\subsection{Geometrical parameters}

The results obtained for the estimation of the porosity $\phi_{s}$, the pore's radius $r_{s}$ and the tortuosity $q_{s}$ are summarized in Tab. 1 for the four materials: a Ceramic Catalyst, a Stainless Steel wire mesh, a nichrome foam and a RVC foam. The comparisons between experiments from Refs. [28, 29] and theoretical (after fitting) T-matrix coefficients are also given in Fig. 3. The uncertainties on the estimated values given in Tab. 1 come from two sources of errors in th eimplementation of the test bench to measure the T-matrices $[28,29]$ : a random error due to noisy microphonic signals and systematic bias errors due to badly known environmental parameters and to the precision of the experimental apparatus itself. The bias error is estimated from the uncertainties on the T-matrix coefficients [28], while the random error is estimated from the Signal to Noise Ratio obtained on each microphone during measurements. However, it is worth noting that the effect of a third source of errors, namely the errors due to all the fixed parameters in the forward problem, cannot be quantified here. As this source of errors is not necessarily small compared with noise and biais, we have to point out that the uncertainties provided in Tab. 1 are probably underestimated.

In the case of the ceramic catalyst, the parameters vector $\Upsilon=\left[\phi_{s} ; r_{s}\right]$ has only two elements because this material is made of straight channels and its tortuosity is fixed to its theoretical ideal value $q_{s}=1$. For this particular case, the hot exchanger and the stack are made of the same material, so that $r_{h}=r_{s}, \phi_{h}=\phi_{s}$ and $q_{h}=q_{s}=1$ [see Eqs. (9)]. The inverse method is thus equivalent to the estimation of the porosity and the pore's radius of a porous sample of length $l_{s}+l_{h}$. The values of porosity and radius obtained from the inverse method are consistent with those expected: the ceramic catalyst being provided by manufacturers with a cell density of 600 CPSI, one can indeed estimate the edge length of one cell, $l_{\text {cell }}$, as

$$
l_{\text {cell }}=\frac{2.54 \cdot 10^{-2}}{\sqrt{600}} \simeq 1 \cdot 10^{-3} \mathrm{~m}
$$

and considering the squared geometry of the channel, the porosity of the material can be given by

$$
\begin{gathered}
\phi_{s}=\left(\frac{2 r_{s}}{l_{\text {cell }}}\right)^{2}, \\
13
\end{gathered}
$$



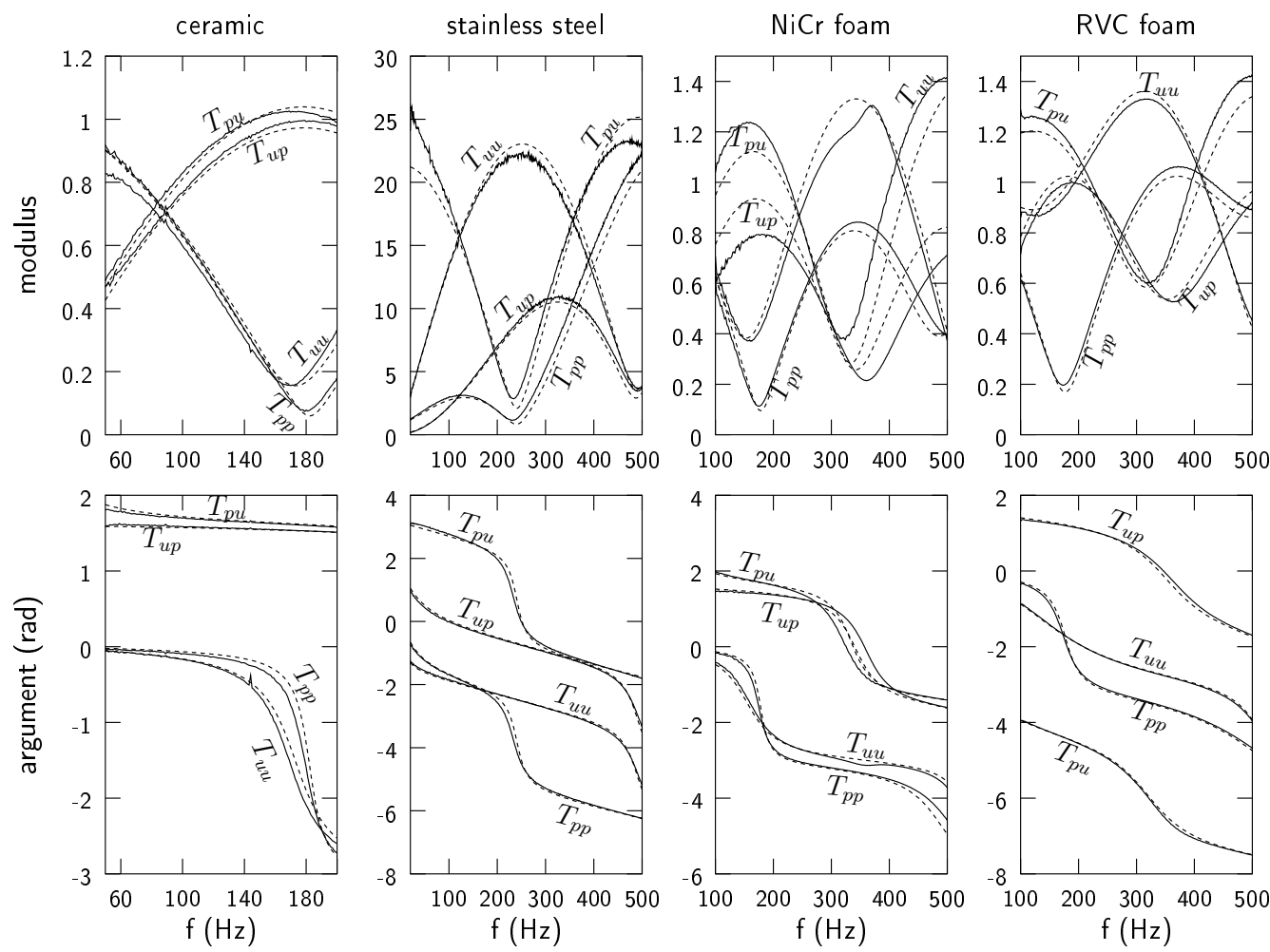

Figure 3: Modulus (upper graphs) and argument (lower graphs) of the four transfer matrix coefficients $\mathcal{T}_{p p}, \mathcal{T}_{p u}, \mathcal{T}_{u p}$ and $\mathcal{T}_{u u}$ as functions of frequency, for the four materials : ceramic sample, stainless steel wire meshes, nichrome foam and RVC foam. The experimental results shown in solid lines come from Refs. [28] and [29]. The dotted lines correspond to the theoretical results obtained after estimation of the parameters $\phi_{s}$ (porosity), $r_{s}$ (average pore's radius) and $q_{s}$ (tortuosity).

which gives, for a porosity $\phi_{s}=0.90$, an equivalent inner radius very close to the estimated value:

$$
r_{s}=\frac{l_{\text {cell }} \sqrt{\phi_{s}}}{2} \simeq 0.49 \mathrm{~mm} .
$$

On the left of Fig. 3 are shown the modulus (upper graph) and argument (lower graph) of the four T-matrix coefficients of the ceramic catalyst, as functions of frequency from 50 to $200 \mathrm{~Hz}$ (corresponding to the range used in experiments). The non-diagonal coefficients $\mathcal{T}_{p u}$ and $\mathcal{T}_{u p}$ are made dimensionless by normalizing them with the characteristic impedance $Z_{c}=\rho c_{0} / S$ of the waveguide. The straight lines represent the experimental results taken from Ref. [28]. The model defined by Eq. (2) and computed with the estimated parameters $\phi_{s}=0.90$ and $r_{s}=0.47 \mathrm{~mm}$ give the results in dashed lines. When looking at these graphs, one can observe that the model fitted with es- 
timated parameters reproduces quite well the experimental transfer coefficients on the whole frequency range. The differences are mainly due to bias errors coming from the measurement method and errors made on fixed environmental parameters such as room temperature, static pressure and accurate location of the elements in the TAC. Ambient heat exchangers can be taken into account with additional T-matrices (additional losses / reflexions) but this does not change significantly the results (though slightly improving them).

For the estimation of the parameters of the three other materials, the porosity and the average radius previously obtained for the ceramic sample are used for the modeling of the hot heat exchanger in Eq. (2) $\left(\phi_{h}=0.90, r_{h}=0.47 \mathrm{~mm}, q_{h}=1\right)$. The results obtained with the inverse method and presented in Tab. 1 are quite close to the manufacturer's data : the Stainless Steel grids are provided with a porosity of 0.45 and a half-edge length of $45 \mu \mathrm{m}$ [37] and the nichrome foam is provided with an average radius of $0.3 \mathrm{~mm}$ (no data about porosity) [38], whereas the RVC foam is provided with a porosity of 0.97 and a cell density of $100 p p i$ corresponding to an average radius of almost $0.13 \mathrm{~mm}$ [39]. The tortuosity of the foams is not provided by the manufacturers and a direct comparison is not possible, as well as for the tortuosity of the Stainless Steel regenerator which is due to a random stacking of the grids.

As for the case of the ceramic catalyst, the Figure 3 present the T-matrix coefficients for these three materials ; the only difference concerns the frequency range which was larger for the experimental characterization of these samples [29]. As for the ceramic sample, the model and the experimental data are in good agreement, but with a larger bias between experiments and fitted theory.

\subsection{Heat exchange coefficients}

When a heat power $Q$ is supplied to the hot heat exchanger, the geometrical parameters $\phi_{s}, r_{s}$ and $q_{s}$ involved in the computation of the forward problem are those obtained with the inverse method in Sect. 5.1. From the results presented in Fig. 3, one can observe that a non-negligible difference between experimental and theoretical data remains after estimation of these geometrical parameters. When the results of the inverse method for $\phi_{s}, r_{s}$ and $q_{s}$ are introduced in the model with a non-zero temperature gradient, this bias is obviously reported in the estimation of the thermal coefficients. In 
order to minimize the effect of this residual bias due to acoustics on the inverse problem associated to heat transfer, the minimization is applied to the "corrected" vector, now defined as

$$
e_{Y, i j}=\bar{Y}_{i}\left(Q_{j}\right) b_{Y, i}-Y_{i}\left(Q_{j}, \mathbf{\Upsilon}\right),
$$

where $b_{Y, i}$ denotes the residual bias:

$$
b_{Y, i}=\frac{Y_{i}(Q=0, \Upsilon)}{\bar{Y}_{i}(Q=0)},
$$

obtained from the acoustical problem.

In the following, the inverse problem is applied to evaluate the heat exchange coefficients between the inside of the TAC and the external walls, namely the parameters $h_{s}, h_{a}$ and $h_{h}$ defined in Eqs. (10). To that purpose, we chose to fix the values $\lambda_{s}, \lambda_{a}$ and $\lambda_{h}$ of the thermal conductivities of the stack, the fluid and the hot heat exchanger, respectively. While the thermal conductivity of air $\lambda_{a}=2.510^{-2} \mathrm{~W} / \mathrm{m} / \mathrm{K}$ (at room temperature) is well known, it might be of interest to determine the thermal conductivities $\lambda_{s}$ and $\lambda_{h}$ from the optimization algorithm as well (i.e. as for the heat exchange coefficients), but however these parameters are too strongly correlated to the heat exchange coefficients so that the inverse problem cannot converge to a unique solution. As a result, the values of $\lambda_{s}$ and $\lambda_{h}$ are obtained from the porosity (determined beforehand) of the stack and the hot heat exchanger, together with an assigned value of the thermal conductivity of the solid material used for the stack or the hot heat exchanger, namely $3 \mathrm{~W} / \mathrm{m} / \mathrm{K}$ for ceramic (cordierite), $13.4 \mathrm{~W} / \mathrm{m} / \mathrm{K}$ for stainless steel, $12 \mathrm{~W} / \mathrm{m} / \mathrm{K}$ for Nichrome, and $6.3 \mathrm{~W} / \mathrm{m} / \mathrm{K}$ for Carbon. It is clear that such an approach has limitation because it does not account for the impact of the tortuosity of the material on heat diffusion along the axis of the TAC, but it was a necessary choice to get information about heat losses towards the walls. As a result, we have to point out that the evaluations of heat exchange coefficients presented in the following should be considered cautiously.

The results obtained for the modulii of the T-matrix after application of the inverse problem are presented in Fig. 4 for the case of the stainless steel grids and for a gradual increase of the heat input from $Q=9 \mathrm{~W}$ up to $Q=81 \mathrm{~W}$. This example is here used to emphasize that adjusting the values of the heat exchange coefficients enables to reproduce experimental data quite well, since the theoretical variations of the T-matrix 

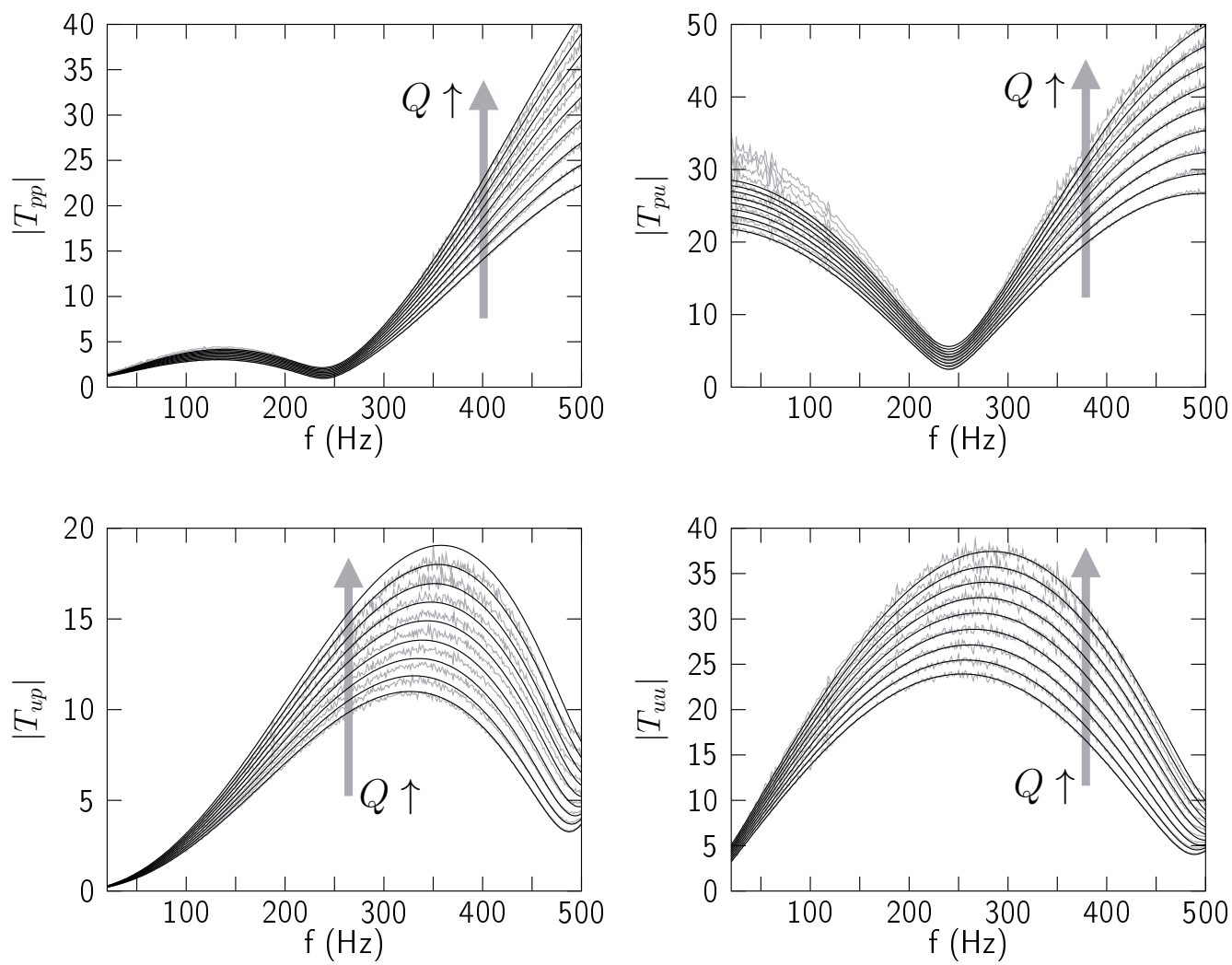

Figure 4: Evolution of the modulus of the acoustical transfer matrix coefficients with the heat power $Q$ for the stainless steel wire meshes. The experimental results shown in grey solid lines come from Ref. [29]. The black lines correspond to the theoretical results obtained after estimation of the heat exchange coefficients.

coefficients as a function of both $\omega$ and $Q$ are almost aligned with their experimental variations. This notably means that the forward model can be used, after application of the optimization process, to predict the scattering of sound by the TAC for some values of heating which were not used in experiments. In the example of Fig. 4 the obtained values for heat exchange coefficients $h_{s} \approx 50 \mathrm{~W} \cdot \mathrm{m}^{-2} \cdot \mathrm{K}^{-1}, h_{h} \approx 40 \mathrm{~W} \cdot \mathrm{m}^{-2} \cdot \mathrm{K}^{-1}$ and $h_{a} \approx 0.2 W \cdot m^{-2} \cdot K^{-1}$ slightly depend on the heat power $Q$, and more details about these variations, as well as those for other stack materials are given in the next figure.

The results of estimations for the three heat exchange coefficients $h_{s}$ (thermoacoustic stack sample), $h_{h}$ (hot exchanger) and $h_{a}$ (thermal buffer tube) as functions of the heat power supply $Q$ and for the four different stack materials are presented in Fig. 5. The range of variations for the heat supply $Q$ departs from $0 \mathrm{~W}$ up to $80 \mathrm{~W}$, except for the 

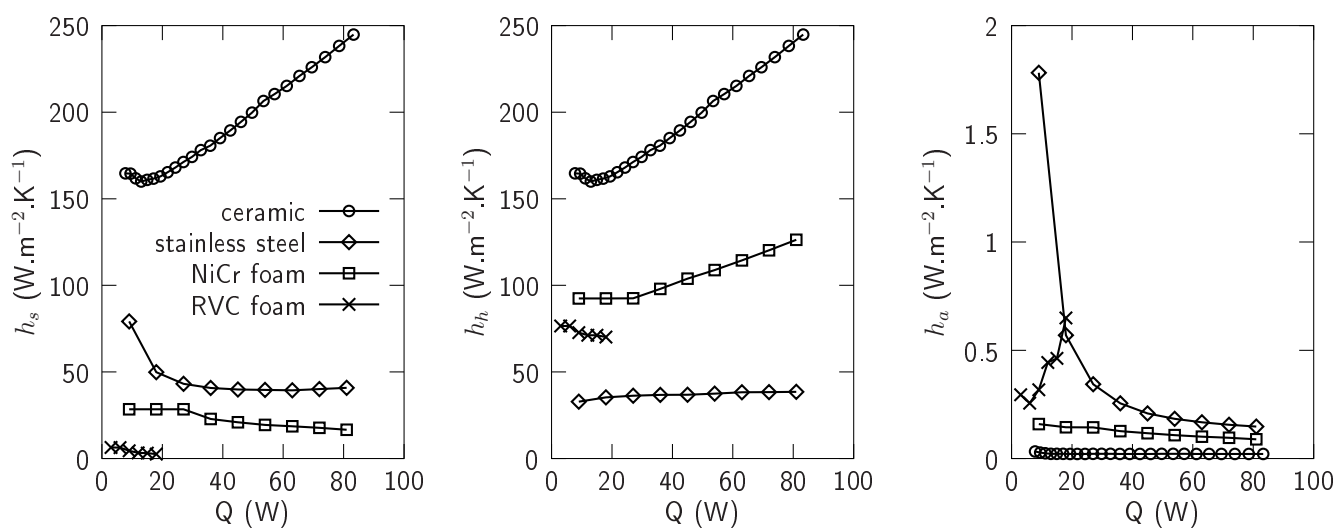

Figure 5: Estimation of the three exchange coefficients $h_{s}$ (thermoacoustic stack sample), $h_{h}$ (hot exchanger) and $h_{a}$ (thermal buffer tube) for the four materials, as function of the heat power $Q$.

case of the RVC foam (risk of combustion for a heat supply higher than $Q=18 W$ ). From the results depicted in Fig. 5 concerning the heat exchange coefficient $h_{s}$ between the stack material and the surrounding walls, one can clearly see that the range of values for $h_{h}$ is large, i.e. from $h_{s} \leq 10 \mathrm{~W} \cdot \mathrm{m}^{-2} \cdot K^{-1}$ for the RVC foam up to $h_{s} \geq 150 \mathrm{~W} \cdot \mathrm{m}^{-2} \cdot \mathrm{K}^{-1}$ for the ceramic catalyst. Such a result is not surprising and can be explained by the differences of the bulk thermal conductivities of the materials, by the geometry of the stack/regenerator itself and the nature of the contact between the material and the inner walls favorizing or not the heat transfer towards the surrounding walls. It is worth noting that the ceramic catalyst, i.e. the only non-tortuous material, exhibits the higher $h_{s}$ coefficient and therefore seems not to be the more adequate material for thermoacoustic applications, since heat leaks towards the walls are not usefull for the process of energy conversion. Another point concerning the results obtained for the estimate of $h_{s}$ is that there exists significant variations (notably for the ceramic catalyst) of this coefficient with the heating $Q$ : such a result can be explained by the variation of thermal conductivities with temperature for both the fluid and the solid frame, and maybe with some variations (dilatation of material?) impacting the thermal contact with the surrounding walls. From the results depicted in Fig. 5 concerning the heat exchange coefficient $h_{h}$ between the hot heat exchanger and the walls, one can see that once again this parameters depends on both the stack material and the heating. Such a result is, however, more surprising because the material used for the hot heat exchanger does not change from one stack 
material to another one: in any case it is made with a Nichrome wire circulating through a ceramic catalyst. These variations could be partly explained by the fact that the length $l_{h}$ of the hot heat exchanger is not the same from one experiment to another: experimental date for ceramic are obtained with $l_{h}=1 \mathrm{~cm}$, while those for stainless steel, and the two foams are obtained with $l_{h}=2.3 \mathrm{~cm}, l_{h}=1.3 \mathrm{~cm}$ and $l_{h}=1.3 \mathrm{~cm}$, respectively. Moreover, $h_{h}$ depends on temperature while the temperature of the hot heat exchnager does not only depend on the heating but also on the ability of the heat exchanger to transmit heat through the stack. Anyway, the range of variations of $h_{h}$ from one series of experiments to another one is not too large (i.e. from $h_{h} \approx 100 \pm 50 W \cdot \mathrm{m}^{-2} \cdot K^{-1}$ ) and finally the results obtained allow to get a rough idea of the numerous sources of errors due to the very simplified description of heat transfer in the forward model. From the results depicted in Fig. 5 concerning the heat exchange coefficient $h_{a}$ in the thermal buffer tube, one can see once again that this heat transfer coefficient depends on the material used for the stack, which should not be the case. However, because this coefficient should depend on temperature, and because the heat transmitted through the TBT also depends on the stack material, the results obtained are not completely surprising.

The resulting temperature distribution along the TAC obtained from the inverse problem are shown in Fig. 6, for the four stack materials and for a heat supply $Q=9 W$. The results obtained clearly show that the choice of a stack material strongly impacts the spatial distribution of the temperature along the entire thermoacoustic core. From the obtained results, one can conclude that the use of a RVC foam allows to get the highest temperature gradient along the stack for a given heat supply, while the ceramic catalysts seem to be worst due to heat leaks through the waveguide walls.

\section{Conclusion}

In this work, the theoretical description of the TAC which has been characterized experimentally in Refs. [28, 29] is presented using a network formalism and a description of the steady-state heat transfer. This model, though simple, can be fitted with experimental data using an inverse method in order to estimate acoustic and thermal parameters of the stack/regenerator placed in the TAC. The inverse problem (minimization problem) is solved iteratively by using a Levenberg-Marquardt derivative method which, 

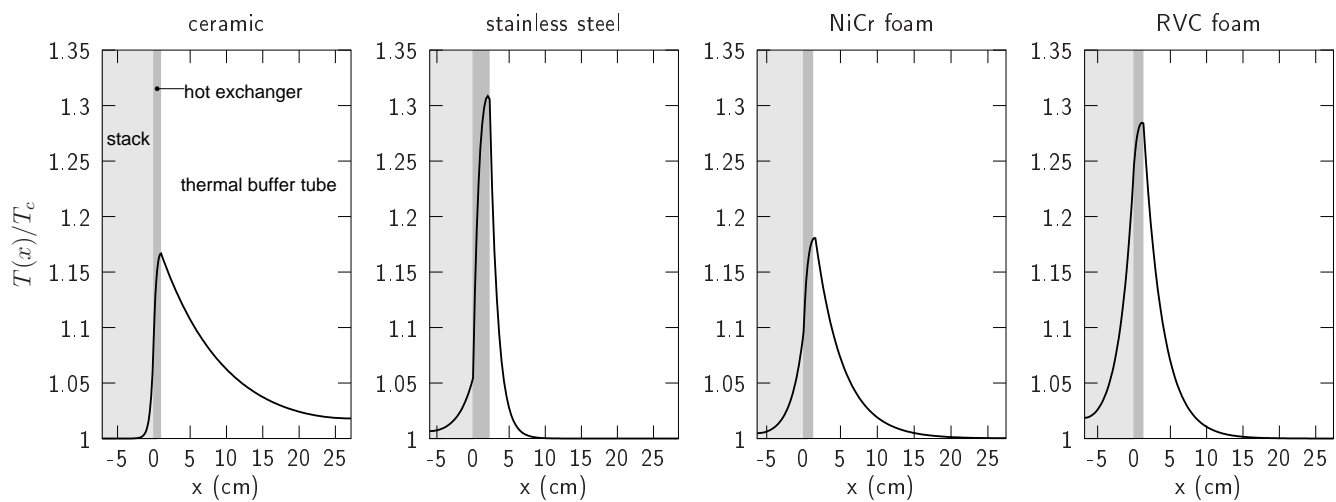

Figure 6: Normalized temperature distribution along the TAC, when equipped with the different samples The heat power is $\mathrm{Q}=9 \mathrm{~W}$. Note that depending on the material used for the experiments, the length of the sample can be slightly different, as well as the one of the ceramic piece used as hot exchanger.

although very sensitive to the choice of parameters, leads to consistent results when an a priori knowledge can be made on their actual values. As a first step, this is applied to the experimental data obtained without heating in order to adjust the porosity and the pore's radius of a squared pores Ceramic Catalyst[28] and to adjust the porosity, radius and tortuosity of more complex materials that can act as a regenerator[29]: a stack of Stainless Steel wire mesh, a Nichrome and a RVC foams. The good agreement between experimental and theoretical curves confirms that our model is consistent and can be used to describe the linear acoustic behaviour of the TAC. As a second step, experimental data are used for estimating heat exchange coefficients characterizing heat transferred laterally towards the surrounding walls. This demonstrates the possibility of estimating thermal parameters of the porous material from acoustic measurements. As expected, the results obtained prove that the heat transfer processes are strongly correlated with the anisotropy of the material. This method thus provides some information about the thermal properties of the stack/regenerator without direct measurements which would require an accurate instrumentation of the cell with thermal sensors. Finally, the estimation of thermal parameters also leads to the quantification of the longitudinal temperature distribution along the TAC which is one of the most important parameters controlling the behaviour of thermoacoustic systems. Although the latter estimates of heat exchange coefficients should be considered cautiously due to the simplistic description of heat transfer used in the forward model, these works confirm 
that a proper design of thermoacoustic engines imply to improve the description of the temperature distribution through the TAC. This temperature distribution is often assumed to be linear along both the stack/regenerator and the thermal buffer tube, while the characterizations of both the mean thermal conductivity and the thermal contact between a porous/tortuous material with its surrounding walls are poorly documented, and poorly understood.

\section{Acknowledgements}

The authors thank Hélène Bailliet, Andrzej Kusiak and Philippe Blanc-Benon for their remarks and suggestions about this work.

[1] S. Backhaus, E. Tward, M. Petach., Traveling-wave thermoacoustic electric generator, Appl. Phys. Lett., $85: 1085,2004$.

[2] G.W. Swift, Thermoacoustics: a unifying perspective for some engines and refrigerators, ed. Acoustical Society of America, Melville, New-York, USA, 2001.

[3] F. Zink, J. S. Vipperman, L. A. Schaefer, Environmental motivation to switch to thermoacoustic refrigeration, Appl. Therm. Eng. 30:119-126, 2010.

[4] M.E.H. Tijani, S. Spoelstra, A hot air driven hermoacoustic-Stirling engine, Appl. Therm. Eng. 61:866-870, 2013.

[5] J.-A. Mumith, C. Makatsoris, T.G. Karayiannis, Design of a thermoacoustic heat engine for low temperature waste heat recovery in food manufacturing: A thermoacoustic device for heat recovery, Appl. Therm. Eng. 65:588-596, 2014.

[6] M. Perier-Muzet, J.-P. Bedecarrats, P. Stouffs, J. Castaing-Lasvignottes, Design and dynamic behaviour of a cold storage system combined with a solar powered thermoacoustic refrigerator, Appl. Therm. Eng. 68:115-124, 2014.

[7] C. Shen, Y. He, Y. Li, H. Ke, D. Zhang, Y. Liu, Performance of solar powered thermoacoustic engine at different tilted angles, Appl. Therm. Eng. 29:2745-2756, 2009.

[8] A.A. Atchley, Standing wave analysis of a thermoacoustic prime mover below onset of self-oscillation, J. Acoust. Soc. Am. 92 (1992) 2907-2914.

[9] A.A. Atchley, H.E. Bass, T.J. Hofler, H.-T. Lin, Study of a thermoacoustic prime mover below onset of self-oscillation, J. Acoust. Soc. Am. 91 (1992) 734-743.

[10] G.W. Swift, Analysis and performance of a large thermoacoustic engine, J. Acoust. Soc. Am. 92 (1992) 1551-1563.

[11] G.W. Swift, R.M. Keolian, Thermoacoustics in pin-array stacks, J. Acoust. Soc. Am. 94 (1993) 941-943.

[12] J.A. Adeff, T.J. Hofler, A.A. Atchley, W.C. Moss, Measurements with reticulated vitreous carbon stacks in thermoacoustic prime movers and refrigerators, J. Acoust. Soc. Am. 104 (1998) 32-38.

[13] S. Backhaus, G.W. Swift, A thermoacoustic Stirling heat engine, Nature 399 (1999) 335-338.

[14] N. Rott, Thermoacoustics, Adv. Appl. Mech. 20 (1980) 135-175.

[15] G.W. Swift, Thermoacoustic engines, J. Acoust. Soc. Am. 84 (1988) 1145-1180.

[16] W.P. Arnott, H.E. Bass, R. Raspet, General formulation of thermoacoustics for stacks having arbitrarily shaped pore cross sections, J. Acoust. Soc. Am. 90 (1991) 3228-3237.

[17] M.R. Stinson, The propagation of plane sound waves in narrow and wide circular tubes, and generalization to uniform tubes of arbitrary cross-sectional shape, J. Acoust. Soc. Am. 89 (1991) 550-558.

[18] H.-S. Roh, W.P. Arnott, J.M. Sabatier, R. Raspet, Measurement and calculation of acoustic propagation constants in arrays of small air-filled rectangular tubes, J. Acoust. Soc. Am. 89 (1991) 2617-2624.

[19] L.A. Wilen, Measurements of thermoacoustic functions for single pores, J. Acoust. Soc. Am. 103 (1998) 1406-1412.

[20] L.A. Wilen, Dynamic measurements of the thermal dissipation function of reticulated vitreous carbon, J. Acoust. Soc. Am. 109 (2001) 179-184. 
[21] A. Petculescu, L.A. Wilen, Lumped-element technique for the measurement of complex density, J. Acoust. Soc. Am. 110 (2001) 1950-1957.

[22] R.T. Muehleisen, C.W. Beamer IV, B.D. Tinianov, Measurements and empirical model of the acoustic properties of reticulated vitreous carbon, J. Acoust. Soc. Am. 117 (2005) 536-544.

[23] M.E. Delany, E.N. Bazley, Acoustical properties of fibrous absorbent materials, Appl. Acoust. 3 (1970) 105-116.

[24] W. Qunli, Empirical relations between acoustical properties and flow resistivity of porous plastic open-cell foam, Appl. Acoust. 25 (1988) 141-148.

[25] J.-F. Allard, P. Herzog, D. Lafarge, M. Tamura, Recent topics concerning the acoustics of fibrous and porous materials, Appl. Acoust. 39 (1993) 3-21.

[26] H.-S. Roh, R. Raspet, H.E. Bass, Parallel capillary-tube-based extension of thermoacoustic theory for random porous media, J. Acoust. Soc. Am. 121 (2007) 1413-1422.

[27] G. Penelet, S. Job, V. Gusev, P. Lotton, M. Bruneau, Dependence of sound amplification on temperature distribution in annular thermoacoustic engines, Acta Acust. United Ac. 91 (2005) $567-577$.

[28] M. Guedra, G. Penelet, P. Lotton, J.-P. Dalmont, Theoretical prediction of the onset of thermoacoustic instability from the experimental transfer matrix of a thermoacoustic core, J. Acoust. Soc. Am. 130 (2011) 145-152.

[29] F.C. Bannwart, G. Penelet, P. Lotton, J.-P. Dalmont, Measurements of the impedance matrix of a thermoacoustic core: applications to the design of thermoacoustic engines, J. Acoust. Soc. Am. 133 (2013) 2650-2660.

[30] M.L. Munjal, Acoustics of ducts and mufflers, ed. Wiley \& Sons, Chichester, UK, 1987.

[31] F.P. Incropera, D.P. DeWitt, Introduction to Heat Transfer, John Wiley \& Sons, New-York, USA, 1985.

[32] G. Penelet, V. Gusev, P. Lotton, M. Bruneau, Experimental and theoretical study of processes leading to steady-state sound in annular thermoacoustic engines, Phys. Rev. E. 72 (2005) 016625.

[33] M.N. Ozisik, Finite Difference Methods in Heat Transfer, CRC Press, USA, 1994.

[34] B. Rémy, S. André, Nonlinear Estimation Problems, Thermal Measurements and Inverse Techniques, CRC Press, USA, 2011. p. 315.

[35] D.W. Marquardt, An algorithm for least-squares estimation of nonlinear parameters, J. Soc. Ind. Appl. Math. 11 (1963) 431-441.

[36] J.V. Beck, K.J. Arnold, Parameters Estimation in Engineering and Science, John Wiley \& Sons, New-York, USA, 1977.

[37] Gantois@ Inox 316L 102083 , www.gantois.fr (last access: 09-12-2014)

[38] RECEMATR RCM-NCAx-2733.10, www.recemat.nl (last access: 09-12-2014)

[39] GoodFellowß LS 364679/1 , www.goodfellow.com (last access: 09-12-2014) 


\section{List of figures}

Fig. 1-. Schematic drawing and photograph of the TAC. Lengths are given in $\mathrm{cm}$.

Fig. 2-. Schematic drawing of the TAC made of four separate media: the air in the thermal buffer tube, the stack/regenerator (considered as an equivalent homogeneous fluid medium), the hot exchanger (considered as an equivalent homogeneous fluid medium) and the stainless steel / aluminium walls.

Fig. 3-. Modulus (upper graphs) and argument (lower graphs) of the four transfer matrix coefficients $\mathcal{T}_{p p}, \mathcal{T}_{p u}, \mathcal{T}_{\text {up }}$ and $\mathcal{T}_{u u}$ as functions of frequency, for the four materials

: ceramic sample, stainless steel wire meshes, nichrome foam and RVC foam. The experimental results shown in solid lines come from Refs. [28] and [29]. The dotted lines correspond to the theoretical results obtained after estimation of the parameters $\phi_{s}$ (porosity), $r_{s}$ (average pore's radius) and $q_{s}$ (tortuosity).

Fig. 4-. Evolution of the modulus of the acoustical transfer matrix coefficients with the heat power $Q$, for the stainless steel wire meshes. The experimental results shown in grey solid lines come from Ref. [29]. The black lines correspond to the theoretical results obtained after estimation of the heat exchange coefficients.

Fig. 5-. Estimation of the three exchange coefficients $h_{s}$ (thermoacoustic stack sample), $h_{h}$ (hot exchanger) and $h_{a}$ (thermal buffer tube) for the four materials, as function of the heat power $Q$.

Fig. 6-. Normalized temperature distribution along the TAC, when equipped with the different samples. The heat power is $\mathrm{Q}=9 \mathrm{~W}$. Note that depending on the material used for the experiments, the length of the sample can be slightly different, as well as the one of the ceramic piece used as hot exchanger. 
Table 1: Estimated values of the geometrical properties of the porous samples. ceramic Stainless Steel NiCr RVC catalyst wire mesh foam foam

\begin{tabular}{lcccc}
\hline \hline$\phi_{s}$ & $0.90 \pm 0.01$ & $0.68 \pm 0.01$ & $0.92 \pm 0.01$ & $0.97 \pm 0.01$ \\
\hline$r_{s}(\mathrm{~mm})$ & $0.47 \pm 0.05$ & $(40 \pm 4) \cdot 10^{-3}$ & $0.31 \pm 0.04$ & $0.17 \pm 0.02$ \\
\hline$q_{s}$ & $1^{a}$ & $1.06 \pm 0.03$ & $1.30 \pm 0.04$ & $1.13 \pm 0.03$ \\
\hline \hline
\end{tabular}

a : fixed parameter, not estimated 